\title{
The Saccharomyces retrotransposon Ty5 integrates preferentially into regions of silent chromatin at the telomeres and mating loci
}

\author{
Sige Zou, Ning Ke, Jin M. Kim, and Daniel F. Voytas ${ }^{1}$ \\ Department of Zoology and Genetics, Iowa State University, Ames, Iowa 50011 USA
}

The nonrandom integration of retrotransposons and retroviruses suggests that chromatin influences target choice. Targeted integration, in turn, likely affects genome organization. In Saccharomyces, native Ty5 retrotransposons are located near telomeres and the silent mating locus HMR. To determine whether this distribution is a consequence of targeted integration, we isolated a transposition-competent Ty5 element from $S$. paradoxus, a species closely related to $S$. cerevisiae. This Ty5 element was used to develop a transposition assay in $S$. cerevisiae to investigate target preference of de novo transposition events. Of 87 independent Ty5 insertions, $\sim 30 \%$ were located on chromosome III, indicating this small chromosome $(\sim 1 / 40$ of the yeast genome) is a highly preferred target. Mapping of the exact location of 19 chromosome III insertions showed that 18 were within or adjacent to transcriptional silencers flanking $H M L$ and $H M R$ or the type $\mathrm{X}$ subtelomeric repeat. We predict Ty5 target preference is attributable to interactions between transposition intermediates and constituents of silent chromatin assembled at these sites. Ty5 target preference extends the link between telomere structure and reverse transcription as carried out by telomerase and Drosophila retrotransposons.

[Key Words: Retrotransposon; Ty5; integration; transposition; silent chromatin; telomere]

Received November 17, 1995; revised version accepted January 19, 1996.

Mobile genetic elements that replicate by reverse transcription are ubiquitous among eukaryotic genomes. These elements, collectively called retroelements, include the retroviruses and two classes of retrotransposons, which are distinguished by whether or not they are flanked by long terminal repeats (LTRs) (Xiong and Eickbush 1990|. A common step in retroelement replication involves the integration of an element's cDNA into the host genome (Brown and Varmus 1989). For the retroviruses and LTR retrotransposons, this step is carried out by a nucleoprotein complex called the integration complex. A key component of the integration complex is the element-encoded integrase protein, which carries out the cutting and joining steps of the integration reaction. Although no specific sequences are required at the target site for integration, the distribution of LTR retroelements is clearly nonrandom (Sandmeyer et al. 1990; Craigie 1992). This suggests that the integration complex is capable of discerning specific features of chromatin to determine sites of integration.

Some of the best examples of retroelement target specificity are provided by the retrotransposons of Saccharo-

${ }^{1}$ Corresponding author. myces cerevisiae. S. cerevisiae has five distinct retrotransposon families, designated Ty1-Ty5, which vary extensively in copy number (from 25 to 30 Tyl/Ty 2 insertions to 0 to 2 Ty5 insertions per haploid genome) (Boeke and Sandmeyer 1991; Zou et al. 1995). A distinctly nonrandom distribution of Tyl-Ty4 insertions has been revealed from the nucleotide sequences of several S. cerevisiae chromosomes, for example, chromosome (chr) III (Oliver et al. 1992; Ji et al. 1993). Most native Tyl-Ty4 elements are found within $1 \mathrm{~kb}$ upstream of genes transcribed by RNA polymerase III (Pol III), such as tRNA genes. For Tyl and Ty3, this genomic organization is the consequence of targeted integration; Pol III genes are the preferred targets of de novo Tyl and Ty3 transposition events /Chalker and Sandmeyer 1990; Chalker and Sandmeyer 1992; Ji et al. 1993; Devine and Boeke 1996).

Mechanisms that dictate Ty target specificity have been studied in detail for Ty3. Ty3 integration is highly precise and typically occurs within the first few base pairs of the start site of Pol III gene transcription (Chalker and Sandmeyer 1992). For tRNA genes, mutations in the promoter that abolish transcription also abolish targeted transposition. Biochemical analyses, including in vitro transposition assays, have demonstrated 
that the Pol III transcription factors TFIIIB and TFIIIC are sufficient for targeted integration (Kirchner et al. 1995). Current models suggest that these transcription factors tether the Ty3 integration complex to its target through protein-protein interactions.

Integration sites for several retroviruses tend to be associated with DNase I hypersensitive sites, suggesting that retroviruses prefer open chromatin (Sandmeyer et al. 1990; Cragie 1992). The yeast two-hybrid system has been used recently to identify a human protein, Inil, that specifically interacts with HIV integrase (Kalpana et al. 1994). Inil is a homolog of the yeast transcription factor SNF5, which is known to remodel chromatin in yeast to promote transcription. The HIV integrase-Inil interaction suggests that retroelements may, in general, recognize specific DNA-bound protein complexes for choosing their integration sites.

In striking contrast to the Tyl-Ty4 families, endogenous Ty5 insertions are located in subtelomeric regions or on chr III adjacent to the silent mating locus $H M R$ (Zou et al. 1995). The telomeres and the transcriptional silencers flanking $H M R$ direct the assembly of a distinct type of chromatin (silent chromatin) that represses the transcription of adjacent genes (Laurenson and Rine 1992). Silent chromatin is also assembled at $H M L$, a second mating locus on chr III, and transcriptional repression of both $H M L$ and $H M R$ prevents the expression of mating type information unless it is copied to MAT, a third, transcriptionally active mating locus. A number of proteins contribute to silent chromatin structure and transcriptional repression at the telomeres and silent mating loci. Among these are proteins involved in DNA replication [the origin recognition complex (ORC); (Bell et al. 1993; Foss et al. 1993; Micklem et al. 1993/], transcription factors [RAPl and ABF1 (Diffley and Stillman 1988; Kurtz and Shore 1991], histones [H3 and H4 (Kayne et al. 1988; Thompson et al. 1994], components of acetyltransferases [NAT1 and ARD1 (Mullen et al. 1989)], and several proteins that have a specific role in silencing [SIR1-SIR4 and RIF1 (Rine and Herskowitz 1987; Hardy et al. 1992)].

Chromatin at the telomeres and silent mating loci is analogous to heterochromatin in other eukaryotes (Hecht et al. 1995). In Drosophila melanogaster, a number of transposable elements are associated with heterochromatin (Pimpinelli et al. 1995), including DNA transposons such as the $\mathrm{P}$ elements, which preferentially transpose to some heterochromatic sites (Karpen and Spradling 1992). Surprisingly, some heterochromatic transposable elements have evolved apparently essential roles for the cell; the $H e T$ and TART retroelements can serve as $D$. melanogaster telomeres (Biessmann et al. 1990; Levis et al. 1993).

In this study we report the isolation of a transpositionally active Ty5 element from the yeast Saccharomyces paradoxus and the development of a Ty5 transposition assay in $S$. cerevisiae. By monitoring the target preference of de novo transposition events, we demonstrate that Ty5 integrates preferentially into regions of silent chromatin on chr III. This suggests that in addition to its well-studied role in transcriptional silencing, silent chromatin serves as a homing device for Ty5 integration. Ty5 target specificity, along with examples of target specificity by elements in other organisms, suggests further that targeted transposition may play a general role in the structure of heterochromatin.

\section{Results}

Identification of full-length Ty5 retrotransposons in $\mathrm{S}$. paradoxus

Many retrotransposon insertions are nonfunctional. An extensive survey of Ty5 elements among diverse $S$. cerevisiae strains failed to identify transposition-competent insertions (Zou et al. 1995). All were either solo LTRs or a degenerate insertion that had accumulated several deleterious mutations (Ty5-1). A survey of other yeast species, however, revealed numerous Ty5 elements in some strains of $S$. paradoxus, the species most closely related to $S$. cerevisiae. This high copy number suggested that these Ty5 elements may be actively transposing. In addition, Ty 5 insertions were found associated with type $\mathrm{X}$ subtelomeric repeats, indicating that their novel genomic organization was conserved between species. Six different Ty5 elements were cloned from one strain (NRRL Y-17217) using internal domain probes from Ty5-1. Preliminary sequencing and restriction mapping identified two structurally similar insertions, Ty5-5p and Ty5-6p (the p denotes their origin from $S$. paradoxus), which were of a size consistent with an active retroelement $(4-6 \mathrm{~kb})$. The complete DNA sequence was obtained for both insertions.

\section{Sequence analysis of Ty5-5p and Ty5-6p}

Ty5-6p is $5376 \mathrm{bp}$ in length and encodes a single open reading frame (ORF) of 1626 amino acids (Fig. 1A). The insertion is flanked by two LTRs of $251 \mathrm{bp}$ that share $>98 \%$ nucleotide identity. A 5-bp direct repeat (TGCTA) is found on either side of Ty5-6p and is likely the target site duplication generated upon transposition. Ty5-6p shares $93.6 \%$ nucleotide identity to the defective $S$. cerevisiae element Ty5-1. The two elements differ primarily by a deletion in Ty5-1 that extends from bases 1475 to 3703 and includes the putative coding region for integrase (see below and Fig. 1A). The boundaries of this deletion are delimited by two 6-bp direct repeats, which may have facilitated loss by recombination.

The Ty5-6p ORF shows significant similarity to proteins encoded by other retrotransposons and retroviruses (Xiong and Eickbush 1990) (Fig. 1B). Near the 5' end is a cysteine motif or finger domain that characterizes LTR retroelement Gag proteins and is thought to be involved in binding RNA. The structure of the Ty5 finger domain, however, is different from those of other retroelements in the spacing of conserved cysteines (typically $\mathrm{CX}_{2} \mathrm{CX}_{4} \mathrm{HX}_{4} \mathrm{C}$ vs. $\mathrm{CX}_{2} \mathrm{CX}_{3} \mathrm{HX}_{4} \mathrm{C}$ for Ty5). The ORF also shows similarity to retroelement Pol proteins, including amino acid sequence domains for protease, integrase, reverse transcriptase, and RNase $\mathrm{H}$. The RT domain shares 
Figure 1. Sequence analysis of $S$. paradoxus Ty5 retrotransposons. (A) A comparison of the structural organization of the $S$. cerevisiae Ty5-1 element and the $S$. paradoxus Ty5-5p and Ty5-6p elements. Open boxes with arrowheads indicate LTRs. Arrows over the internal domain indicate ORFs, and arrowheads denote stop codons. Stippled and hatched boxes depict conserved amino acid sequence domains: (RB), RNA binding; (PR), protease; (IN), integrase; (RT), reverse transcriptase; (RH), RNase $H$. The lines designated $a$ and $b$ under Ty5-6p mark the probes used in Fig. 2. The percentages reflect nucleotide identity between elements. The naturally occurring deletion in Ty5-1 is noted by the broken line, and the Ty5-1 RB domain is unrecognizable because of nucleotide changes. Bases in Ty5-5p that differ from Ty5-6p are noted below the element. $(B)$ Amino acid sequence alignments of domains conserved between Ty5-6p and other retrotransposons. Sequences used in the alignment are from closely related Tyl-copia group retrotransposons, including Tyl $\mathrm{H} 3$ from $S$. cerevisiae (M18706), Tal-3 from Arabidopsis thaliana (X13291) and copia from D. melanogaster (M11240). Black boxes denote identical amino acids shared among at least three of the four aligned elements. Numbers indicate amino acid residues omitted. Note: Tyl does not encode an RNA binding domain. $(C)$ The Ty5 primer binding site is complementary to the anticodon stem-loop of $S$. cerevisiae initiator methionine tRNA. (O) Nucleotides that pair to PBSs; (O) nonpaired nucleotides. Nucleotides 63-76 are complementary to the Tyl PBS; nucleotides 69-76 are complementary to the Ty3 PBS; nucleotides $28-40$ are complementary to the putative Ty5 PBS. This latter region is shown aligned to the Ty5-1 and Ty5-6p PBSs. The sequences of other retrotransposon PBSs that begin at the same nucleotide position are shown. GenBank accession numbers for these sequences are Osser, X69552; copia, M11240; and Tp2, X52770. Nucleotide differences between some PBSs may reflect sequence differences among host initiator tRNAs or element mutations.

the highest amino acid similarity to the $D$. melanogaster copia element. This similarity, and the fact that IN precedes RT, clearly place Ty5 within the Tyl-copia group elements, a class of LTR retrotransposons found in the genomes of diverse eukaryotes.

The DNA sequence of Ty5-5p differed from Ty5-6p by six nucleotide substitutions and a single $A$ insertion /Fig. 1A). Four of the six substitutions reside in the last $150 \mathrm{bp}$ of the 3' LTR and lie outside the Ty5-5p ORF. One of the internal domain substitutions is silent (base 897), and the other (base 1186) results in an amino acid change from Cys in Ty5-6p to Phe in Ty5-5p. This change may affect protease function, as it is in the predicted active site for protease. The single $\mathrm{A}$ insertion occurs in a stretch of $\mathrm{A}_{7}$ and creates a frameshift before the RT domain. Frameshifts immediately before RT have not been observed in other retroelements, and this mutation likely renders Ty5-5p nonfunctional. It is important to note that neither Ty5-5p nor Ty5-6p have a frameshift or stop codon at the junction of their Gag- and Pol-like domains [i.e., in the vicinity of protease (Fig. 1A)]. This

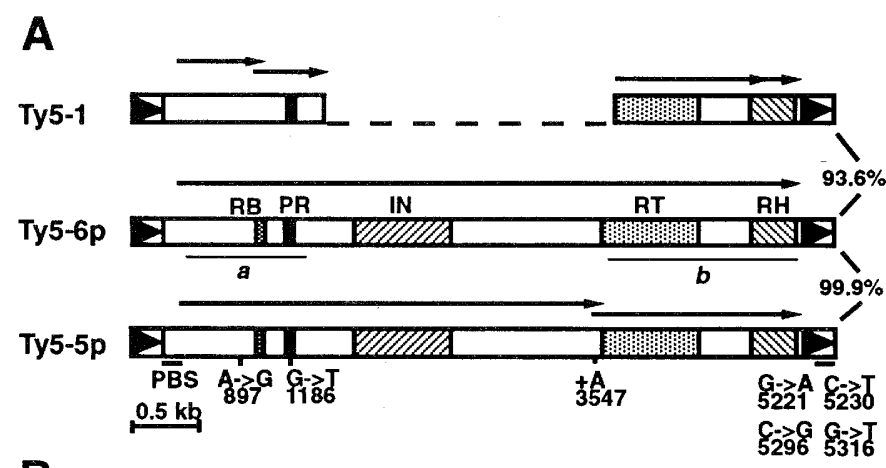

B

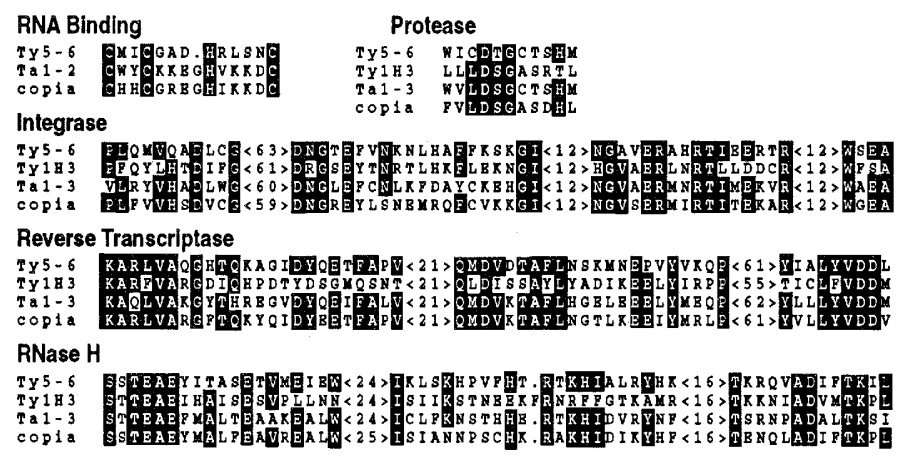

C

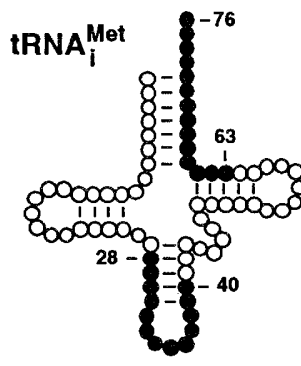

genomic organization is unusual for retroelements and has been observed only for several related Tyl-copia group retrotransposons, including copia and several plant elements (e.g., Mount and Rubin 1985; Voytas and Ausubel 1988), as well as the more distantly related Tf1 element from Schizosaccharomyces pombe (Levin et al. 1990).

Immediately adjacent to the $5^{\prime}$ LTR of both $S$. paradoxus and S. cerevisiae Ty5 elements are 13 nucleotides that are complementary to the anticodon stem-loop of the $S$. cerevisiae initiator methionine tRNA /Figs. 1C and 2 C). For most LTR retrotransposons and retroviruses (including the other four families of Ty elements), this region constitutes the primer binding site (PBS) and is complementary to the $3^{\prime}$ end of a host tRNA. Base-pairing between the PBS and a host tRNA is used to prime DNA synthesis during reverse transcription. Several retrotransposon families have been identified with PBSs similar to Ty5, and complementarity to the host initiator methionine tRNA begins at the exact same position in the anticodon stem (Fig. 1C). 


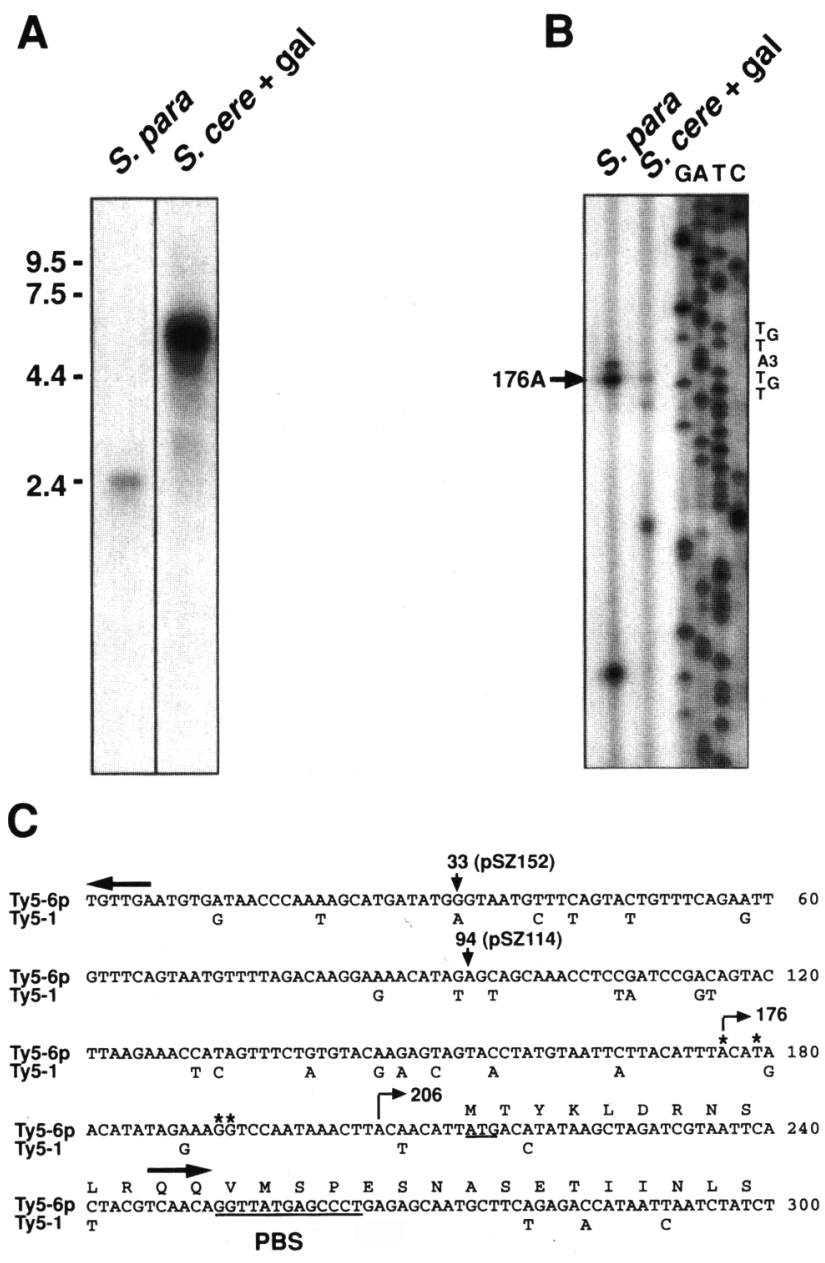

Figure 2. Analysis of Ty5-6p transcripts. (A) Northern analysis of Ty5 transcripts. The lane designated $S$. para shows hybridization of probe $a$ (Fig. 1A) to $0.7 \mu \mathrm{g}$ of poly(A) RNA isolated from log-phase cultures of S. paradoxus strain NRRL Y17217. The 2.4-kb transcript was also observed with probe $b$ (data not shown). The lane marked $S$. cere + gal shows hybridization of probe $a$ to $15 \mu \mathrm{g}$ of total RNA from $S$. cerevisiae cells carrying plasmid pSZ152 that had been grown on $2 \%$ galactose for $24 \mathrm{hr}$. The transcript corresponds in size to the full-length Ty5-6p element, including the his $A I$ marker gene. Note: The intensity of hybridization does not reflect relative expression levels. $(B)$ Primer extension analysis of RNAs described in $A$. The sequencing ladder was generated with a subclone of the Ty5-6p 5' LTR with the same primer used for primer extensions. Base positions of the major start sites of transcription are noted. $(C)$ Sequence features of the Ty5-6p 5' LTR. Bases that differ from the $S$. cerevisiae Ty5-1 element are shown below the sequence. Arrows denote the terminal inverted repeats. Hooked arrows indicate the transcription start sites of Ty5 in S. paradoxus; asterisks indicate start sites in $S$. cerevisiae determined using galactose-induced mRNA. Vertical arrows mark the positions of the GAL1-10 UAS fusions. The PBS is underlined, as is the ATG, which marks the beginning of the Ty5 ORF.

\section{Transcription and transcript mapping of Ty5}

The high degree of nucleotide similarity between Ty5-6p and Ty5-1 suggested that the $S$. paradoxus retrotransposons might replicate in $S$. cerevisiae. This would per- mit the use of genetic and molecular tools available in $S$. cerevisiae, including the extensive genome sequence, to study Ty5 target specificity. Successful transposition assays developed for Tyl and Ty3 have used heterologous promoters to control transcription and achieve high levels of regulated transposition (Boeke et al. 1985; Chalker and Sandmeyer 1990|. In such assays it was important to maintain the structure of the native mRNA, because it is used as a template for both protein synthesis and reverse transcription.

Northern analysis of poly (A) mRNA prepared from $S$. paradoxus revealed a single low abundance transcript of $2.4 \mathrm{~kb}$, which does not correspond in size to the predicted full-length transcript $(\sim 5 \mathrm{~kb})$ (Fig. 2A). Shorter transcripts generated by splicing have been observed for the closely related $D$. melanogaster copia elements. In this instance, differential splicing is used to regulate the stoichiometry of $\mathrm{Gag}$ and Gag-Pol gene products (Yoshioka et al. 1990). The 2.4-kb Ty5 mRNA, however, hybridizes to probes corresponding to both the Gag- and Pol-like regions of Ty5, suggesting that it is not the product of a similar splicing event (Figs. 1A and 2A; data not shown). In addition, the well-conserved splice-site consensus sequences are not found in Ty5-6 (Rymond and Rosbash 19921. The 2.4-kb mRNA may therefore be the product of a deleted Ty5 insertion.

Transcription of Ty5-6p was placed under galactose control by replacing part of the 5' LTR with a GAL1-10 upstream activation sequence (UAS). Two different constructs were generated, which differed by the site of the GAL1-10 UAS fusion (base 33, pSZ152 and base 94, pSZ114; Fig. 2Cl. Only pSZ152 generated high levels of galactose-induced message as indicated by Northern analysis (Fig. 2A). A single mRNA was detected, which corresponds to the expected full-size Ty5 transcript; no smaller mRNAs were observed with probes corresponding to either the $5^{\prime}$ or $3^{\prime}$ regions of the Ty5 ORF (Fig. 2A; data not shown|. Both the galactose-induced message and the 2.4-kb mRNA from $S$. paradoxus were used to map transcription start sites by primer extension. For the $S$. paradoxus message, major start sites were identified at bases 176 and 206 within the 5' LTR (Fig. 2B,C). The start site at base 176 was shared with the galactose-induced Ty5 message from pSZ152 (Fig. 2B); this construct is transpositionally active (see below), suggesting that base 176 is the actual start site of the Ty5 genomic mRNA.

\section{Ty5-6p transposes in $\mathrm{S}$. cerevisiae}

The galactose-inducible Ty5-6p construct was modified to facilitate detection of transposition by incorporating a selectable marker gene between the end of the Ty5-6p ORF and the beginning of the 3' LTR (Fig. 3A). A HIS3 marker gene designed to specifically detect Tyl transposition by reverse transcription was used (Curcio and Garfinkel 1991). The HIS3 gene carries an artificial intron (AI) inserted into the HIS3 coding sequence in the antisense orientation, which blocks HIS3 gene expression. The his $3 A I$ marker, however, is oriented in Ty5-6p 

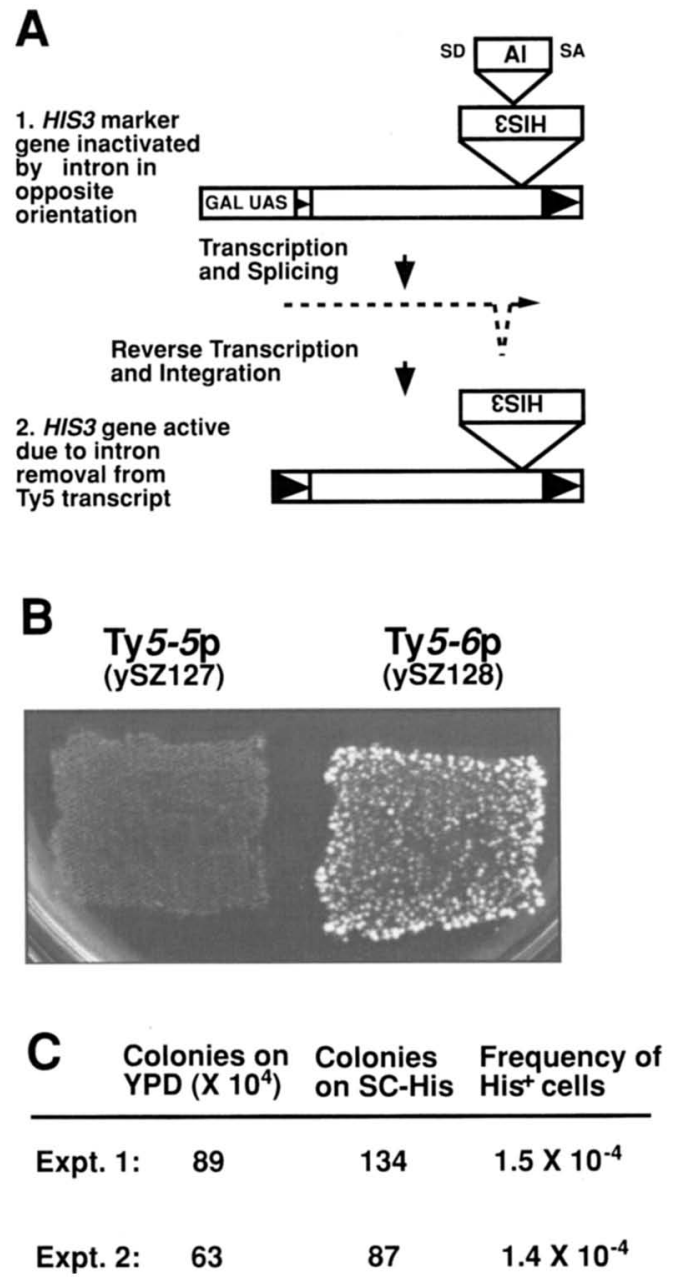

Figure 3. A Ty5 transposition assay in $S$. cerevisiae. (A) Selection scheme for Ty5 transposition. The Ty5-5p and Ty5-6p elements were placed under the transcriptional control of the GAL1-10 UAS and modified further by insertion of a his $3 A I$ marker gene between the end of the Ty5 ORF and the beginning of the $3^{\prime}$ LTR. In this marker, the HIS3 gene is interrupted by an artificial intron (AI) that is in the antisense orientation relative to the HIS3 message. (SD) Splice donor; (SA) splice acceptor (step 1). The intron, however, is in the sense orientation to be spliced from the Ty5 transcript and the HIS3 gene can be reconstituted after reverse transcription of the spliced Ty 5 message (step 2). (B) Yeast cell patches carrying Ty5-5p (ySZ127) and Ty5-6p (ySZ128) were grown on galactose-containing media to induce transcription and replica plated to media lacking histidine to select for the reconstituted HIS3 gene. His ${ }^{+}$cells were only observed for Ty5-6p constructs. (C) Frequency of His ${ }^{+}$cells in ySZ128 carrying Ty5-6p. Cells were scraped from patches on galactose-containing media, diluted in $\mathrm{dH}_{2} \mathrm{O}$, and plated on rich media (YPD) and synthetic media without histidine ( $\mathrm{SC}-\mathrm{H}$ ). Colonies were counted after growth at $30^{\circ} \mathrm{C}$, and the frequency was calculated by dividing the colony number on $\mathrm{SC}-\mathrm{H}$ plates by the product of the colony number on YPD plates and the dilution factor.

such that the intron is on the sense strand of the retrotransposon. The intron, therefore, can be spliced from the Ty5-6p transcript, and a functional HIS3 gene can be generated through reverse transcription and integration into the genome. A similar construct was also generated using Ty $5-5 \mathrm{p}$, which has a naturally occurring frameshift mutation before RT.

Patches of $S$. cerevisiae cells carrying the two Ty5 constructs were grown on galactose-containing media to induce transcription and transposition and then replica plated to media lacking histidine to select for putative transposition events (Fig. 3B). $\mathrm{His}^{+}$cells arose only from patches containing the Ty5-6p construct. The frequency of $\mathrm{His}^{+}$cells after $48 \mathrm{hr}$ of galactose induction was approximately $1.5 \times 10^{-4}$ (Fig. 3C). The absence of $\mathrm{His}^{+}$ cells from the Ty5-5p construct suggested that no functional reverse transcriptase was synthesized, likely because of the frameshift mutation and/or the amino acid substitution in protease. The $\mathrm{His}^{+}$phenotype was dependent on galactose induction (data not shown), indicating a requirement for Ty5-6p transcription.

The reconstituted HIS 3 gene generated by reverse transcription could integrate into the genome either by transposition or recombination. These two events can be distinguished, however, because transposition generates target site duplications. Southern hybridization analysis of four independent $\mathrm{His}^{+}$strains revealed that each carried a Ty 5 insertion on a uniquely sized restriction fragment, suggesting that the marked Ty5-6p had integrated into different locations in the genome (Fig. 4A). One putative transposition event was cloned, and the sequences flanking the Ty5 element were determined. These sequences matched the sequence of chr III near the $H M R$ locus (Oliver et al. 1992). Moreover, a 5-bp direct repeat immediately flanking the element was generated upon integration, as this 5 -bp sequence is present as a single copy on chr III (Fig. 4B). Interestingly, the integration site is near $H M R-E$, the cis-acting sequences required for transcriptional silencing at $H M R$ (for review, see Laurenson and Rine 1992). More specifically, this insertion is $442 \mathrm{bp}$ from the autonomously replicating consensus sequence (ACS) in $H M R-E$, which serves as an origin of replication. The presence of a target site duplication indicates that Ty5-6p is transpositionally competent, and the site of integration supports the hypothesis that silent chromatin is a preferred target for Ty5.

\section{Ty5-6p transposes preferentially to chr III}

To investigate Ty5 target preference more thoroughly, chromosomes from 148 independent $\mathrm{His}^{+}$strains were separated on pulsed-field gels and transferred to nylon membranes. Of these strains, 61 (41\%) had multiple chromosomes that hybridized strongly with HIS3 probes, indicating multiple Ty5 insertions. Data describing the genomic organization of these elements will be presented elsewhere. Of the 148 strains, 87 (59\%) had single chromosomes carrying Ty 5 insertions (Table 1). Of these 87 strains, $26(-30 \%)$ had insertions located on chr III. Southern hybridization analysis performed on 22 of these strains indicated that each had a single Ty5 insertion (data not shown). If the size of the chromosome 
A

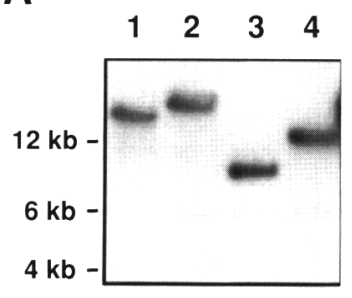

B

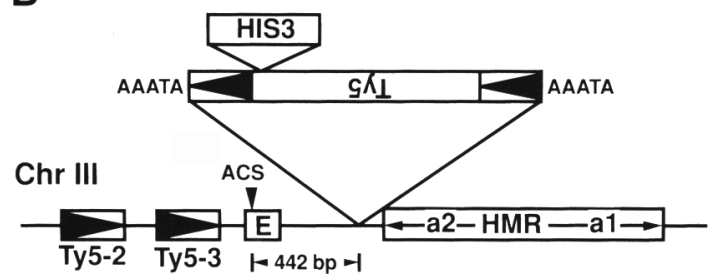

Figure 4. Physical analysis of de novo Ty5-6p transposition. (A) Southern hybridization analysis of independent $\mathrm{His}^{+}$ strains. Genomic DNAs were prepared from four $\mathrm{His}^{+}$strains that had lost the parental pSZ152 plasmid after selection on 5-FOA. DNA was digested with Xhol, which does not cut within Ty5-6p and hybridized with a HIS3 probe, which does not hybridize to the original strain. Each strain carries a HIS3 gene on a uniquely sized XhoI fragment that is larger than the 6.5-kb plasmid-borne Ty5-6p construct, consistent with transposition of a Ty5 element. $|B|$ The site of insertion of a de novo Ty5 transposition event at the HMR locus on chr III. The HMR locus is depicted as an open box, with the $a 1$ and $a 2$ genes noted by arrows. E denotes the transcriptional silencer of $H M R$, and the arrowhead marks the ACS within HMR-E. The site of Ty5 integration is 442 bases from this ACS. The 5 bases shown on either side of the element are the target site duplications generated upon integration. Upstream of $H M R-E$ are two native solo Ty5 LTRs.

targets are taken into account, the chr III insertions occurred at a relative density of approximately one per 12 $\mathrm{kb}$. This density is more than 4 times greater than the density observed for chrs I and VI, 7 times greater than that observed for chr XI, and at least 10 times greater than that observed for all other chromosomes.

\section{Ty5-6p transposes preferentially to the telomeres and silent mating loci on chr III}

The four native Ty5 insertions on chr III and the one de novo transposition event described above are all located near the left telomere or the HMR-E transcriptional silencer. To determine whether the genomic organization of Ty5 elements is a consequence of preferential integration at these sites, the polymerase chain reaction (PCR) was used to map the exact location of de novo Ty5 insertions in the collection of strains carrying chr III insertions. Sequences flanking these elements were amplified by either the inverse PCR (Ochman et al. 1988) or standard PCR using Ty5-specific oligonucleotides and oligonucleotides that flanked potential target sites, namely the telomeres and the $\mathrm{E}$ and I transcriptional silencers at $H M L$ and $H M R$. PCR products were sequenced directly and used to map precisely the target site of integration by comparison to the chr III sequence (Oliver et al. 1992). Sequences were determined for 19 of the 26 independent chr III insertions. Flanking sequences on both sides of four insertions were determined and shown to have 5-bp target duplications, indicating they arose by transposition (data not shown). No consensus sequence can be deduced from all known Ty5 target sites, suggesting that Ty5 integration is sequence independent.

One Ty5 element (W147) is located near a hot spot for Tyl transposition (Fig. 5; Table 2). This insertion site is $>5 \mathrm{~kb}$ from the nearest tRNA gene (SUF16); most de novo Tyl insertions at this site are within $700 \mathrm{bp}$ of this tRNA (Ji et al. 1993; Devine and Boeke 1996). Eighteen of the elements, however, were clustered near cis-acting sequences required for the assembly of silent chromatin. These included four insertions near the left telomere (W2, W55, W77, W84), three of which were located within $900 \mathrm{bp}$ of the end of chr III. In addition, three insertions were located near the transcriptional silencer $H M L-E$ (W7, W28, W144), four near HML-I (W68, W79, W134, W114), four near HMR-E (W9, W66, W76, W113), and three near HMR-I (W27, W44, W51). No insertions were found near the right telomere; this may be attributable to inefficient PCR amplification of sequences in this region. Previously, we have used the ACSs located within the transcriptional silencers and the telomeric $\mathrm{X}$ repeats as reference points to orient native Ty5 insertions (Zou et al. 1995). De novo Ty5 insertions in silent chromatin are distributed on either side of these ACSs. Twelve of the 18 insertions occurred within $600 \mathrm{bp}$ of an ACS, and all are located within $1.3 \mathrm{~kb}$. Nine of the elements are oriented toward and eight away from the nearest ACS, indicating no apparent orientation specificity.

\section{Discussion}

Sequence of Ty5-6p reveals unique features

A S. paradoxus Ty5 element $($ Ty5-6p) was identified that shares $>95 \%$ nucleotide identity with the previously characterized, nonfunctional $S$. cerevisiae element

Table 1. The distribution of 87 independent Ty5 transposition events among S. cerevisiae chromosomes

\begin{tabular}{lcc}
\hline Chromosomes & $\begin{array}{l}\text { Number of } \\
\text { insertions }\end{array}$ & $\begin{array}{l}\text { Density of } \\
\text { insertions per kb }\end{array}$ \\
\hline III & 26 & $1 / 12$ \\
I/VI & 10 & $1 / 51$ \\
IX & 5 & $1 / 88$ \\
XI & 5 & $1 / 133$ \\
II/X & 11 & $1 / 140$ \\
VII/XV & 14 & $1 / 164$ \\
IV & 7 & $1 / 234$ \\
XVI/XIII & 8 & $1 / 244$ \\
V/VIII & 1 & $1 / 1100$ \\
\hline
\end{tabular}


Figure 5. Distribution of Ty5 transposition events on chr III. The shaded bar indicates chr III. Expanded views are shown for the left telomere, HML and HMR. Locations of de novo Ty5 integrations are indicated by the short vertical arrows and strain designation. Arrows pointing down indicate insertions in the same $5^{\prime} \rightarrow 3^{\prime}$ orientation as the chr III sequence. Arrows pointing upward represent insertions in the opposite orientation. The positions of relevant $A C S s$ are indicated by long vertical arrows. Native Ty5 elements are shown (Ty5-1, Ty5-2, Ty5-3, Ty5-4). For the left telomere, $\mathrm{T}$ designates the $\mathrm{TG}_{(1-3)}$ repeats, and $\mathrm{X}$ is the subtelomeric repeat. For $H M L$ and $H M R, E$ and $I$ designate the flanking transcriptional silencers. $W, X$, and $Z$ represent homologous regions shared between $H M L, H M R$, and $M A T$. Ya and Ya are specific for $H M L$ and $H M R$, respectively. Transcripts $(\mathbf{a} 1, \mathbf{a} 2, \alpha 1, \alpha 2)$ at each mating locus are shown by horizontal arrows.

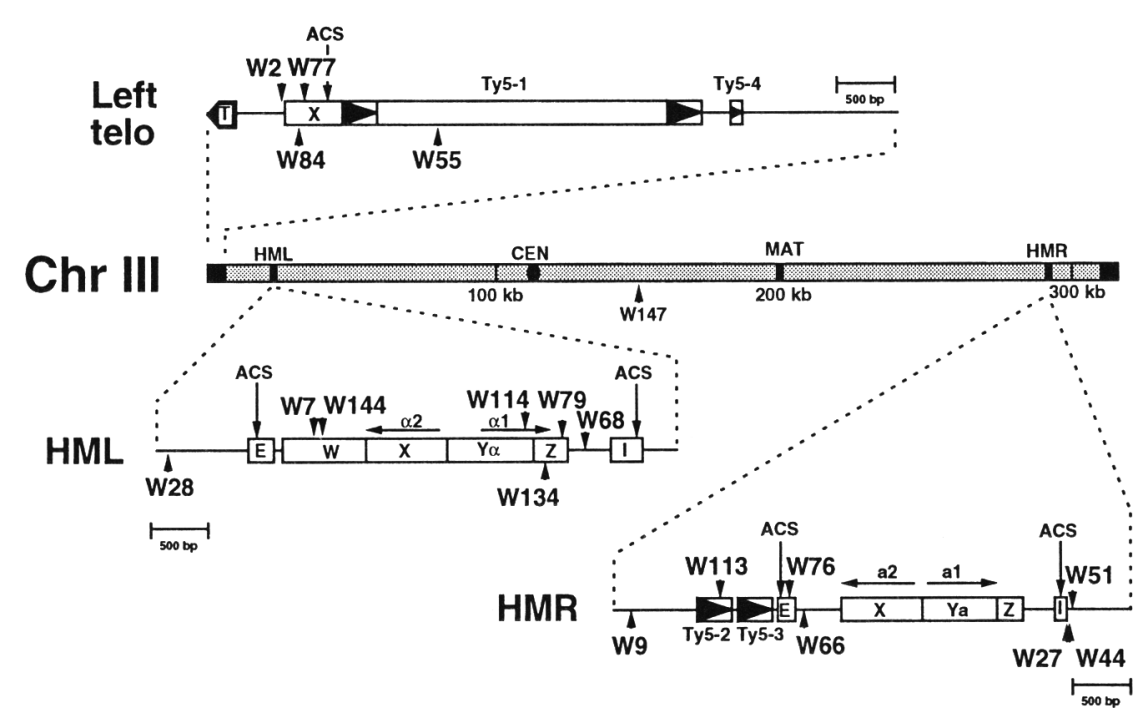

Ty5-1 (Voytas and Boeke 1992). The genomic organization of Ty5-6p and sequence similarities between its reverse transcriptase and those of other retrotransposons indicate that it is a member of the Tyl-copia group elements (Xiong and Eickbush 1990). Ty5-6p, however, has two novel features that distinguish it from previously characterized yeast Tyl-copia group elements (Tyl, Ty2, and Ty4) (Boeke and Sandmeyer 1991). First, the Ty5-6p Gag and Pol genes are encoded within a single ORF. In most retrotransposons and retroviruses, these two genes are differentially expressed, typically because of translational frameshifting or readthrough (Jacks 1990). The related D. melanogaster copia elements also encode a single ORF and use differential mRNA splicing to regulate Gag and Pol expression (Yoshioka et al. 1990). A single full-length mRNA was detected from the transpositionally active Ty5-6p element in $S$. cerevisiae, however, suggesting that Ty5 utilizes a different regulatory mechanism. The $\mathrm{Tf} 1$ element of $S$. pombe is transcribed as a single mRNA and also encodes a single ORF (Levin et al. 1990). Gag/Pol stoichiometry of $T f 1$ is regulated by differential protein stability (Atwood et al. 1996); it will

Table 2. Locations of de novo Ty5 transposition events on chr III

\begin{tabular}{llccc}
\hline $\begin{array}{l}\text { Ty5.insertion } \\
\text { number }\end{array}$ & Locus & $\begin{array}{l}\text { Nucleotide position } \\
\text { on chr III }\end{array}$ & $\begin{array}{l}\text { Target site } \\
\text { sequence }\end{array}$ & $\begin{array}{c}\text { Distance to } \\
\text { closest ACS }\end{array}$ \\
\hline W2 & left telomere & 625 & G A A A C & 413 \\
W84 & left telomere & 798 & C T C A C & 240 \\
W77 & left telomere & 853 & T A T A C & 185 \\
W55 & left telomere & $1842 \pm 50$ & N.D. & $850 \pm 50$ \\
W28 & $H M L-E$ & 10499 & A T T A C & 747 \\
W7 & $H M L-E$ & 11786 & T A T T T & 531 \\
W144 & $H M L-E$ & 11797 & C C A T G & 542 \\
W114 & $H M L-I$ & 13584 & G T T T G & 1104 \\
W134 & $H M L-I$ & 13783 & C A T T T & 905 \\
W79 & $H M L-I$ & 13911 & T A T A C & 788 \\
W68 & $H M L-I$ & 14164 & T G T T C & 535 \\
W147 & Tyl hot spot & $147535 \pm 50$ & N.D. & N.D. \\
W9 & $H M R-E$ & 290076 & C T T A C & 1291 \\
W113 & $H M R-E$ & 290843 & A A T T C & 525 \\
W76 & $H M R-E$ & 291467 & G A A A G & 87 \\
W66 & $H M R-E$ & 291541 & G A A T T & 58 \\
W44 & $H M R-I$ & G G A A T & 57 \\
W27 & $H M R-I$ & G T T G G & 106 \\
W51 & $H M R-I$ & 293675 & & \\
\hline
\end{tabular}

a(N.D.) Not determined. 
be of interest to determine whether Ty5 exploits a similar strategy.

The second unusual feature of Ty5 is the PBS for reverse transcription, which is complementary to the anticodon stem-loop of the $S$. cerevisiae initiator methionine tRNA. This unusual PBS is also found in copia and a few related retrotransposons from diverse organisms (Voytas and Boeke 1993). For the copia elements, the initiator methionine tRNA is cleaved in the anticodon stem-loop by an unknown mechanism to generate the primer used to initiate reverse transcription (Kikuchi et al. 1986). This novel priming mechanism may now be amenable to genetic analysis in yeast using Ty5 and resources developed previously to study the role of the initiator tRNA in priming Tyl reverse transcription (Chapman et al. 1992; Keeney et al. 1995).

\section{Ty5-6p is transposition competent}

A Ty5 transposition assay was developed for S. cerevisiae by placing Ty5-6p under the transcriptional control of the GAL1-10 promoter. This assay exploited a his $3 A I$ marker gene to select for replication by reverse transcription (Curcio and Garfinkel 1991). Histidine prototrophs were selected after induction of Ty5-6p transcription, and a newly transposed element was cloned. This insertion arose by transposition, as it was immediately flanked by 5-bp duplications of target DNA generated upon integration. The promoter of the HIS3 marker in this insertion was within $750 \mathrm{bp}$ downstream of the transcriptional silencer at $H M R \cdot E$, which can repress transcription of some genes as far away as $2.3 \mathrm{~kb}$ (Brand et al. 1985). It has been shown previously, however, that HIS3 is not effectively silenced when inserted at $H M R$ (D. Shore, pers. comm.). Strains with HIS3 inserted at $H M R$ grow in the absence of histidine, although a weak silencing effect has not been excluded. This observation is also supported by the fact that we were able to use HIS 3 to identify Ty5 insertions throughout other silent regions on chr III (see below). In addition, silencing is not simply disrupted by the insertion of Ty5. Two insertions at HML-E (W7, W144) (Fig. 5) are mating competent, indicating that $H M L$ is still repressed (data not shown). Nonetheless, we cannot formally exclude the possibility that some transposition events went undetected because of the requirement of our assay for HIS3 expression.

Approximately $30 \%$ of the strains tested carried multiple Ty5 insertions on different chromosomes. These elements appear to be organized in arrays (S. Zou, N. Ke and D.F. Voytas, unpubl.). Arrays of Tyl have been observed (Weinstock et al. 1990), particularly in Tyl integrase mutants, and these arrays are likely generated by recombination of cDNA into preexisting genomic Ty1 copies (Sharon et al. 1994). We have observed lower rates of Ty5 transposition in recombination-deficient (rad52) hosts, suggesting that recombination is responsible for generating some His ${ }^{+}$cells (N. Ke and D.F. Voytas, unpubl.). In this study we have therefore restricted our analysis to $\mathrm{His}^{+}$strains that have single Ty5 insertions. In all cases examined, the presence of flanking target site duplications indicated that these insertions were generated by transposition rather than recombination.

\section{Silent chromatin is a target for Ty5}

$H M L$ and $H M R$ encode genes that specify yeast mating type. These genes are not expressed until they are transferred to a third locus $(M A T)$ in the middle of chr III by directed gene conversion. Transcriptional repression of $H M L$ and $H M R$ is mediated by proteins that assemble at flanking cis-acting sequences, designated the $\mathrm{E}$ and I silencers (Laurenson and Rine 1992). Similar to the $H M$ loci, the telomeres are also transcriptionally silent (telomere position effect) (Gottschling et al. 1990). Many proteins that play a role in silencing the $H M$ loci also act at the telomeres and are required for telomere position effect (Fig. 6). Some of these proteins bind the subtelomeric X repeat (Longtine et al. 1993), which is found at

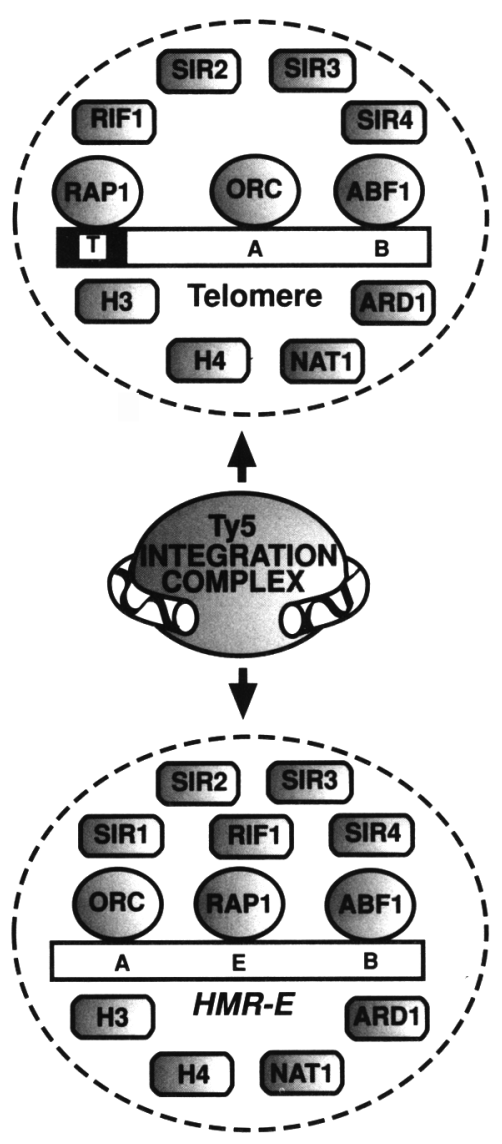

Figure 6. Ty5 targets and components of silent chromatin. A, $\mathrm{E}$ and $\mathrm{B}$ denote protein binding domains at the transcriptional silencer $H M R-E$, the telomere, and the subtelomeric $\mathrm{X}$ repeat (for review, see Laurenson and Rine 1992). A is an ACS, which is bound by the ORC. E denotes RAP1-binding sites, which are the $\mathrm{TG}_{(1-3)}$ repeats at the telomere and sequence $5^{\prime}$-ACCCATTCATAA-3' at HMR-E (note that the E domain is a component of the $H M R-E$ silencer). B denotes ABFl-binding sites. Other regulators that act at these sites are shown schematically above the DNA-bound proteins. 
the ends of all chromosomes (Louis et al. 1994). Although $\mathrm{X}$ repeats are not required for silencing per se (Gottschling et al. 1990), they are highly conserved in $S$. paradoxus, suggesting they may serve a role in telomere structure (Zou et al. 1995).

We characterized in detail de novo Ty5 insertions on chr III, which represented $\sim 30 \%$ of the transposition events in strains containing single insertions. Eighteen of $19 \mathrm{chr}$ III insertions were near or within the cis-acting sequences that assemble silent chromatin. Using the ACS as a reference point, 12 insertions were clustered in either orientation within $600 \mathrm{bp}$ of ACSs located in the subtelomeric $\mathrm{X}$ repeat or the $\mathrm{E}$ and I regulatory regions; the remainder were within $1.3 \mathrm{~kb}$ of these ACSs. The proximity of Ty 5 elements to silent chromatin is not limited to the chr III insertions. Target sites for several Ty5 elements on other chromosomes were found to reside in telomeric regions (J. Kim and D.F. Voytas, unpubl.).

Silent chromatin may turn off Ty5 transcription after integration. Ty5 transcripts were undetectable in strains with Ty5 insertions near HMR (S. Zou and D.F. Voytas, unpubl.). Transcriptional silencing is also a feature of regions flanking Pol III genes, preferred targets for Tyl and Ty3 integration. Pol III transcription strongly inhibits expression of adjacent RNA Pol II promoters (Hull et al. 1994). Although silencing near Pol III genes may occur by a different mechanism than that at the telomeres and silent mating loci, transcriptional silencing may be a general means by which retrotransposition in yeast is regulated.

Most Ty5 integration events occurred in regions shown previously to occlude sequence-specific DNAbinding proteins such as restriction endonucleases (Loo and Rine 1994). For example, sites for cleavage by $H O$ endonuclease /which is involved in mating type switching) are found at all three mating loci, but cleavage is blocked at $H M L$ and $H M R$ by silent chromatin (Laurenson and Rine 1992). It has long been known that during mating type switching, MATa cells preferentially recombine with $H M L \alpha$, and MAT $\alpha$ cells preferentially recombine with HMRa (for review, see Haber 1992). The mechanism that determines this donor preference appears to be transparent to Ty5, as insertions occur at equal frequencies at both silent mating loci in either MATa or $M A T \alpha$ strains (S. Zou and D.F. Voytas, unpubl.). It appears the Ty5 integration complex can overcome barriers imposed by silent chromatin, or transposition may occur only when these regions are readily accessible, such as shortly after DNA replication.

Based on the strong bias for sites of Ty5 insertion, we predict that targets are chosen through interactions between Ty 5 proteins (such as integrase) and some component of silent chromatin (Fig. 6). Similar models have been put forth to explain the preference of Ty3 for genes transcribed by RNA Pol III (Kirchner et al. 1995). The transcription factors TFIIIB and TFIIIC, which interact with the tRNA promoter, are likely candidates for determining Ty3 target specificity. Tyl also frequently integrates upstream of Pol III transcribed genes; however, the pattern of integration is considerably less precise and occurs over a 700-bp region upstream of target genes (Ji et al. 1993; Devine and Boeke 1996). This lack of precision is also a hallmark of Ty5 transposition. No specific component of silent chromatin can be implicated as the interacting partner based on the pattern of Ty5 insertion sites. The genetic resources available from the extensive study of transcriptional silencing in S. cerevisiae should facilitate the identification of interacting partners.

Other retroelements may also sense specific DNAbound protein complexes to determine sites of integration. Recently, two-hybrid screens with HIV integrase have identified a specific interacting protein, Inil, which is a homolog of the yeast transcription factor SNF5 (Kalpana et al. 1994). Understanding how integration complexes identify their target DNAs may suggest new means by which retroelements can be directed to specific sites of the genome. This may have direct applications for improving the site selectivity of retroviral vectors used for gene therapy.

\section{Ty5 as a class of subtelomeric repeats}

The silent mating loci and telomeres of $S$. cerevisiae share many features with heterochromatin found in other eukaryotes, including late replication in S phase, association with the nuclear envelope, and epigenetic gene repression (Roth 1995). Another interesting feature of heterochromatin is the association with transposable elements. For example, transposable elements are a major structural component of $D$. melanogaster heterochromatin (Karpen and Spradling 1992; Pimpinelli et al. 1995). Heterochromatin may offer a "safe haven" for elements to integrate without negative genetic consequences for the host such as deleterious mutations in essential genes (Ji et al. 1993). Ty5 target preference suggests that the link between transposition and heterochromatin may be widespread.

Transposable elements associated with telomeric heterochromatin have evolved important cellular roles in some organisms. For example, in D. melanogaster, two non-LTR retrotransposons, HeT and TART, can transpose preferentially to the ends of chromosomes and serve as telomeres (Biessmann et al. 1990; Levis et al. 1993). In $S$. cerevisiae, another class of telomeric repeats, the $\mathrm{Y}^{\prime}$ elements have structural features reminiscent of transposable elements (Louis et al. 1994). Y' elements have never been demonstrated to transpose, but they may be remnants of telomere-specific transposons that are no longer functional. Amplification of $\mathrm{Y}^{\prime}$ elements by recombination at the telomeres can suppress telomerelength mutations, such as est 1 (Lundblad and Blackburn 1993). Telomerase-independent elongation of telomeres has also been observed in some human cancer cell lines (Bryan et al. 1995). With the preference for Ty5 to integrate at telomeres, it will be of interest to determine whether Ty 5 amplification by transposition and/or recombination can also suppress telomere mutations. The fact that telomerase is a reverse transcriptase indicates the importance of reverse transcription in maintaining 
chromosome structure. The discovery of Ty5 and its novel target preference adds to the growing list of reverse transcribing elements that influence the structure of chromosome ends.

\section{Materials and methods}

\section{Strains}

The yeast strains used in this study are as follows: $S$. paradoxus NRRL Y-17217 (Northern Regional Research Laboratory), GRF167 (MATa his3D200 ura3-167) (J.D. Boeke, Johns Hopkins University School of Medicine, Baltimore, MD), W303-1A (MATa ade2-1 can1-100 his3-11 leu2-3 trp1-1 ura3-1) (A. Myers, Iowa State University). The Escherichia coli strain XL1-blue was used for recombinant DNA manipulations. Transformation of $E$. coli and yeast strains was performed by electroporation as described (Ausubel et al. 1987).

\section{Endogenous Ty5 isolation and sequence determination}

The construction and screening of the $S$. paradoxus genomic DNA libraries containing Ty5 elements has been described previously (Zou et al. 1995). Templates for DNA sequencing were generated by $\gamma \delta$ mutagenesis of Ty5-5p and Ty5-6p subclones (Gold BioTechnology, Inc.). DNA sequence was obtained using the fmol sequencing kit (Promega) or by the ISU Nucleic Acid Facility. Sequence analysis was performed using the Genetics Computer Group programs (Devereux et al. 1984). Sequences were determined for one DNA strand of Ty5-5p and both strands of Ty5-6p.

\section{Analysis of Ty5 transcription}

Transcription of Ty5 was monitored by Northern analysis (Ausubel et al. 1987). Filters were prepared with $0.7 \mu \mathrm{g}$ of poly(A) mRNA isolated from S. paradoxus strain NRRL Y17217 or with $15 \mu \mathrm{g}$ of total mRNA from GRF167 carrying plasmid pSZ152 (ySZ128) (see below). Hybridizations were conducted with probes corresponding to the Gag- and Pol-like domains of Ty5-6p (Fig. 1A) that had been radiolabeled by random priming (Promega). The transcriptional start site of Ty5 was mapped by primer extension with the primer DVo207 (5'-AGGGCTCATAACCTGTTGAC-3') (Ausubel et al. 1987).

\section{Ty5 transposition construct}

To develop a Ty5 transposition assay, the Ty5-6p element was first modified to regulate its transcription by galactose. Specifically, the GAL1-10 UAS was PCR-amplified from plasmid pJEF1105 (gift from J.D. Boeke) with primers DVol84 (5'-TCTCGAGCCCCATTATCTTAGC-3') and DVo185 (5'-CGTCGACTCATCCTATGGTTGTT-3' $\%$ The PCR amplicon was then digested with $X h o I$ and Sall and subcloned into the XhoI site of the URA3-based $2 \mu$ plasmid pRS426 (Stratagene), to yield pSZ138. Ty5-6p sequences from position 33 to 443 were amplified from a Ty5-6p HindIII subclone with primer DVol82 (5'GGGTAATGTTTCAGT-3') and the universal primer. This PCR product was subcloned into the PCR cloning vector pT7Blue (Novagen) to create pSZ135, which has a SalI site upstream of position 33 in Ty5-6p. A Sall-HindIII fragment from pSZ135 was subcloned into the SalI-HindIII sites of pSZ138 to yield pSZ139, which placed the GAL1-10 UAS upstream of position 33 in the Ty5-6p 5' LTR.

To select Ty5-6p transposition events, a marker gene was inserted after the Ty5 ORF and before the 3' LTR. To generate appropriate restriction sites, the 3 ' end of Ty5-6p was amplified by two pairs of primers: DVo187 (5'-CGGTACCTATATACCAC-3'), DVol89 (5'-GAGATCTGTTATTTTGCAGTTTCT$\left.3^{\prime}\right)$ and DVol88 (5'-CAGATCTCATGCGTATTCAGTT-3'), DVo190 (5'-TGGATCCTGTTGACGTAGTGAATTA-3). Both PCR amplicons were sequentially subcloned into the KpnI$X$ hoI and BamHI sites in pRS426 to yield pSZ128. This resulted in an insertion of polylinker sites, including a ClaI site at position 5099 in Ty5-6p. Both XhoI and BamHI sites in the pRS426 polylinker were destroyed during cloning. A BamHI-KpnI fragment from pSZ128 and a HindIII-KpnI fragment from the internal sequence of Ty5-6p were subcloned into the BamHI-HindIII sites in pSZ139 by three-way ligation to yield pSZ147. Finally a ClaI fragment with the his $3 A I$ marker from pGTymhis $3 A I$ was inserted to the ClaI site in pSZ147 (Curcio and Garfinkel 1991). A ligation product with the his $3 A I$ gene in the antisense orientation to Ty5-6p was chosen as the final version of the Ty5 transposition construct, pSZ152. A Ty5-5p transposition construct was generated by replacing the Ty5-6p internal HindIIIKpnI sequence with that of Ty5-5p to yield pSZ151. All PCR products were confirmed by DNA sequencing.

\section{Ty5 transposition assay}

S. cerevisiae strain GRF167 was transformed with plasmid pSZ151 and pSZ152 by electroporation to yield ySZ127 and ySZ128 (Ausubel et al. 1987). Transposition assays were performed with modifications as described (Keeney et al. 1995). Cells were grown as patches on $\mathrm{SC}-\mathrm{U} /$ glucose plates at $30^{\circ} \mathrm{C}$ for 2 days. The patches were subsequently replica plated to $\mathrm{SC}-\mathrm{U} /$ galactose medium and grown for an additional 2 days at $23^{\circ} \mathrm{C}$. Finally, the patches were replicated to SC media without histidine $(\mathrm{SC}-\mathrm{H})$ and incubated for $\sim 60 \mathrm{hr}$ to select $\mathrm{His}^{+}$cells. To quantitate the rate of $\mathrm{His}^{+}$cell formation, cells were scraped from patches on $\mathrm{SC}-\mathrm{U} /$ galactose plates after 2 days growth and suspended in $10 \mathrm{ml}$ of $\mathrm{dH}_{2} \mathrm{O}$. Subsequently, $200 \mu \mathrm{l}$ of the cell suspension and $200 \mu \mathrm{l}$ of a $10^{-4}$ cell dilution were plated on $\mathrm{SC}-\mathrm{H}$ and rich media (YPD) plates, respectively. Colonies were counted after growth at $30^{\circ} \mathrm{C}$ for $60 \mathrm{hr}$. The frequency of His ${ }^{+}$ cells was calculated by dividing the colony number on $\mathrm{SC}-\mathrm{H}$ plates by the product of the colony number on YPD plates and the dilution factor.

To physically characterize de novo Ty5-6p insertions, $\mathrm{His}^{+}$ cells resulting from transposition assays were grown on 5-fluoro-orotic acid (5-FOA) plates to lose the URA3-based plasmid pSZ152 (Ausubel et al. 1987). Genomic DNA was isolated from four $\mathrm{His}^{+} / \mathrm{Ura}^{-}$strains. Approximately $1 \mu \mathrm{g}$ of this DNA was digested with XhoI, separated on $0.8 \%$ agarose gels, and transferred to nylon membranes by the alkaline method (Ausubel et al. 1987). Filters were hybridized with ${ }^{32}$ P-labeled HIS3 sequences to identify candidate transposition events. One such event was cloned by screening a partial genomic library constructed from a His ${ }^{+} / \mathrm{Ura}^{-}$strain by methods described previously (Zou et al. 1995). Flanking sequences from both sides of the Ty5-6p insertion were determined with oligonucleotides DVo200 (5'-CATTACCCATATCATGCT-3') and DVo183 (5'CCTCGAGCAGCAAACCTCCGA-3').

\section{Chromosome analysis of Ty5 insertions}

To investigate the chromosome distribution of de novo Ty5-6p insertions, 148 individual $\mathrm{His}^{+} / \mathrm{Ura}^{-}$colonies were recovered from transposition assays with ySZ135 (W303-1A carrying pSZ152). Chromosomes were prepared in low melting agarose plugs, separated in pulsed-field gels using standard conditions 
(BioRad), transferred to nylon membranes, and hybridized with a ${ }^{32}$ P-labeled HIS3-specific probe.

\section{Mapping Ty5 insertions onto chr III}

Genomic DNA was isolated from W303-1A strains with a Ty5 insertion on chr III. For nine strains, sequences flanking the Ty5 insertion were determined by inverse PCR. Approximately 100 ng of genomic DNA was digested with the restriction enzyme $M s p I$ in $20 \mu l$. The digestion products were then ligated in $50 \mu 1$ at $15^{\circ} \mathrm{C}$ overnight to promote self-ligation. The ligation mixture $(2 \mu \mathrm{l})$ was amplified with the Ty5-6p LTR-specific oligonucleotides DVo219 (5-'TACTGTCGGATCGGAGGTTT-3') and DVo220 (5'-CTGTGTACAAGAGTAGTACC-3'). For the remaining strains, sequences flanking the Ty5 insertions were amplified by standard PCR. LTR-specific primers (DVo219 or DVo220) were used in conjunction with oligonucleotides flanking the transcriptional silencer HMR-E, HMR-I, HML-E, and $H M L-I$, as well as chr III sub-telomeric $\mathrm{X}$ repeats. These latter oligonucleotides were DVo211 (5'-TGGTAGAAGCAGTAGTAACT -3 ') and DVo212 (5' -ACCAGAGAGTGTAACAACAG$\left.3^{\prime}\right)$ for HMR-E; DVo251 (5'-TGCTGAAGTACGTGGTGAC-3') and DVo253 (5'-AGCCCTATTCGCGTCGTG-3') for HMR-I; DVo247 (5'-CACGAGCTCATCTAGAGCC-3') or DVo250 (5'GCCTCTCCTTCTAAGAAGAT-3') and DVo212 for $H M L-E$; and DVo251 and DVo252 (5'-TTCTCGAAGTAAGCATCAAC$\left.3^{\prime}\right)$ for HML-I. Note that DVo212 and DVo251 are complementary to sequences shared by $H M R, H M L$, and MAT. The oligonucleotides DVo213 [5'-TAGAATATTTTTATGTTTAG/G/ C)TGA(T/G)TTT-3'] and DV0254 [5'-AAA/C/A|TCA/C/ G|CTAAACATAAAAATATTCTA-3'] match the ACSs in both the left and right chr III subtelomeric $X$ repeats. Sequences flanking Ty5 insertions were determined with oligonucleotides DVo214 (5'-CCCTCGAGCATTTACATAACATATAGAAAG$\left.3^{\prime}\right)$ or DVo243 (5'-CCTTGTCTAAAACATTACTG-3').

\section{Acknowledgments}

We are grateful to Jef Boeke, Scott Devine, Drena Dobbs, Tom Peterson, and Steven Rodermel for critical reading of the manuscript. We also thank Jef Boeke and Alan Myers for strains and plasmids and Phil Farabaugh for helpful comments regarding sequence analysis. This work was funded by a grant from the American Cancer Society and an American Cancer Society Junior Faculty Research Award to D.F.V. This is journal paper no. $\mathrm{J}-16592$ of the Iowa Agriculture and Home Economics Experiment Station, Ames, IA (project no. 3120).

The publication costs of this article were defrayed in part by payment of page charges. This article must therefore be hereby marked "advertisement" in accordance with 18 USC section 1734 solely to indicate this fact.

\section{Note}

The GenBank accession numbers for the sequences reported in this paper are U19263 and U19264.

\section{References}

Atwood, A., J. Lin, and H.L. Levin. 1996. The retrotransposon Tfl 1 assembles VLPs that contain excess gag relative to integrase because of a regulated degradation process. Mol. Cell. Biol. 16: 338-346.

Ausubel, F.M., R. Brent, R.E. Kingston, D.D. Moore, J.G. Seidman, J.A. Smith, and K. Struhl. 1987. Current protocols in molecular biology. Greene/Wiley Interscience, New York.

Bell, S.P., R. Kobayashi, and B. Stillman. 1993. Yeast origin recognition complex functions in transcription silencing and DNA replication. Science 262: 1844-1849.

Biessmann, H., J.M. Mason, K. Ferry, M. d'Hulst, K. Valgeirsdottir, K.L. Traverse, and M.L. Pardue. 1990. Addition of telomere-associated HeT DNA sequences "heals" broken chromosome ends in Drosophila. Cell 61: 663-673.

Boeke, J.D., and S.B. Sandmeyer. 1991. Yeast transposable elements. In The Molecular and cellular biology of the yeast Saccharomyces (ed. J.R. Broach, J.R. Pringle, and E.W. Jones), pp. 193-261. Cold Spring Harbor Laboratory Press, Cold Spring Harbor, NY.

Boeke, J.D., D.J. Garfinkel, C.A. Style, and G.R. Fink. 1985. Ty elements transpose through an RNA intermediate. Cell 40: 491-500.

Brand, A.H., L. Breeden, J. Abraham, R. Sternglanz, and K. Nasmyth. 1985. Characterization of a "silencer" in yeast: A DNA sequence with properties opposite to those of a transcriptional enhancer. Cell 41: 41-48.

Brown, P. and H. Varmus. 1989. Retroviruses. In Mobile DNA (ed. D.E. Berg and M.M. Howe), pp. 53-108. American Society for Microbiology, Washington, D.C.

Bryan, T.M., A. Englezou, J. Gupta, S. Bacchetti, and R.R. Reddel. 1995. Telomere elongation in immortal human cells without detectable telomerase activity. EMBO J. 14: 42404248.

Chalker, D.L. and S.B. Sandmeyer. 1990. Transfer RNA genes are genomic targets for de novo transposition of the yeast retrotransposon Ty3. Genetics 126: 837-850.

- 1992. Ty3 integrates within the region of RNA polymerase III transcription initiation. Genes \& Dev. 6: 117-128.

Chapman, K.B., A.S. Bystrom, and J.D. Boeke. 1992. Initiator methionine tRNA is essential for Tyl transposition in yeast. Proc. Natl. Acad. Sci. 89: 3236-3240.

Craigie, R. 1992. Hotspots and warm spots: integration specificity of retroelements. Trends Genet. 8: 187-190.

Curcio, M.J. and D.J. Garfinkel. 1991. Single-step selection for Tyl element retrotransposition. Proc. Natl. Acad. Sci. 88: $936-940$.

Devereux, J., P. Haeberli, and O. Smithies. 1984. A comprehensive set of sequence analysis programs for the VAX. Nucleic Acids Res. 12: 387-395.

Devine, S.E. and J.D. Boeke. 1996. Integration of the yeast retrotransposon Tyl is targeted to regions upstream of genes transcribed by RNA polymerase III. Genes \& Dev. (this issue).

Diffley, J.F.X. and B. Stillman. 1988. Purification of a yeast protein that binds to origins of DNA replication and a transcriptional silencer. Proc. Natl. Acad. Sci. 85: 2120-2124.

Foss, M., F.J. McNally, P. Laurenson, and J. Rine. 1993. Origin recognition complex (ORC) in transcriptional silencing and DNA replication in S. cerevisiae. Science 262: 1838-1844.

Gottschling, D.E., O.M. Aparicio, B.L. Billington, and V.A. Zakian. 1990. Position effect at $S$. cerevisiae telomeres: reversible repression of Pol II transcription. Cell 63: 751-762.

Haber, J.E. 1992. Mating-type gene switching in Saccharomyces cerevisiae. Trends Genet. 8: 446-452.

Hardy, C.F., L. Sussel, and D. Shore. 1992. A RAPl-interacting protein involved in transcriptional silencing and telomere length regulation. Genes \& Dev. 6: 801-814.

Hecht, A., T. Laroche, S. Strahl-Bolsinger, S.M. Gasser, and M. Grunstein. 1995. Histone $\mathrm{H} 3$ and $\mathrm{H} 4 \mathrm{~N}$-termini interact with SIR3 and SIR4 proteins: A molecular model for the formation of heterochromatin in yeast. Cell 80: 583-592. 
Hull, M.W., J. Erickson, M. Johnston, and D.R. Engelke. 1994. tRNA genes as transcriptional repressor elements. Mol. Cell. Biol. 14: 1266-1277.

Jacks, T. 1990. Translational suppression in gene expression in retroviruses and retrotransposons. Curr. Topics Microbiol. Immunol. 157: 93-124.

Ji, H., D.P. Moore, M.A. Blomberg, L.T. Braiterman, D.F. Voytas, G. Natsoulis, and J.D. Boeke. 1993. Hotspots for unselected Ty1 transposition events on yeast chromosome $I I I$ are near tRNA genes and LTR sequences. Cell 73: 1007-1018.

Kalpana, G.V., S. Marmon, W. Wang, G.R. Crabtree, and S.P. Goff. 1994. Binding and stimulation of HIV-1 integrase by a human homolog of yeast transcription factor SNF5. Science 266: 2002-2006.

Karpen, G.H. and A.C. Spradling. 1992. Analysis of subtelomeric heterochromatin in the Drosophila minichromosome Dp1 187 by single P element insertional mutagenesis. Genetics 132: 737-753.

Kayne, P.S., U.J. Kim, M. Han, J.R. Mullen, F. Yoshizaki, and M. Grunstein. 1988. Extremely conserved histone H4 N terminus is dispensable for growth but essential for repressing the silent mating loci in yeast. Cell 55: 27-39.

Keeney, J.B., K.B. Chapman, V. Lauermann, D.F. Voytas, S.U. Astrom, U. von Pawel-Rammingen, A. Bystrom, and J.D. Boeke. 1995. Multiple molecular determinants for retrotransposition in a primer tRNA. Mol. Cell. Biol. 15: 217226.

Kikuchi, Y., Y. Ando, and T. Shiba. 1986. Unusual priming mechanism of RNA-directed DNA synthesis in copia retrovirus-like particles of Drosophila. Nature 323: 824-826.

Kirchner, I., C.M. Connolly, and S.B. Sandmeyer. 1995. Requirement of RNA polymerase III transcription factors for in vitro postion-specific integration of a retroviruslike element. Science 267: 1488-1491.

Kurtz, S. and D. Shore. 1991. RAP1 protein activates and silences transcription of mating-type genes in yeast. Genes $\&$ Dev. 5: 616-628.

Laurenson, P. and J. Rine. 1992. Silencers, silencing, and heritable transcriptional states. Microbiol. Rev. 56: 543-560.

Levin, H.L., D.C. Weaver, and J.D. Boeke. 1990. Two related families of retrotransposons from Schizosaccharomyces pombe. Mol. Cell. Biol. 10: 6791-6798.

Levis, R.W., R. Ganesan, K. Houtchens, L.A. Tolar, and F.M. Sheen. 1993. Transposons in place of telomeric repeats at a Drosophila telomere. Cell 75: 1083-1093.

Longtine, M.S., S. Enomoto, S.L. Finstad, and J. Berman. 1993. Telomere-mediated plasmid segregation in Saccharomyces cerevisiae involves gene products required for transcriptional repression at silencers and telomeres. Genetics 133: $171-182$.

Loo, S. and J. Rine. 1994. Silencers and domains of generalized repression. Science 264: 1768-1771.

Louis, E.J., E.S. Naumova, A. Lee, G. Naumov, and J.E. Haber. 1994. The chromosome end in yeast: its mosaic nature and influence on recombinational dynamics. Genetics 136: 789802.

Lundblad, V. and E.H. Blackburn. 1993. An alternative pathway for yeast telomere maintenance rescues est 1 - senescence. Cell 73: 347-360.

Micklem, G., A. Rowley, J. Harwood, K. Nasmyth, and J.F. Diffley. 1993. Yeast origin recognition complex is involved in DNA replication and transcriptional silencing. Nature 366: 87-89.

Mount, S.M. and G.M. Rubin. 1985. Complete nucleotide sequence of the Drosophila transposable element copia: ho- mology between copia and retroviral proteins. Mol. Cell. Biol. 5: 1630-1638.

Mullen, J.R., P.S. Kayne, R.P. Moerschell, S. Tsunasawa, M. Gribskov, M. Colavito-Shepanski, M. Grunstein, F. Sherman, and R. Sternglanz. 1989. Identification and characterization of genes and mutants for an $\mathrm{N}$-terminal acetyltransferase from yeast. EMBO J. 8: 2067-2075.

Ochman, H., A.S. Gerber, and D.L. Hartl. 1988. Genetic applications of an inverse polymerase chain reaction. Genetics 120: 621-623.

Oliver, S.G., Q.J.M. van der Aart, M.L. Agostoni-Carbone, M. Aigle, L. Alberghina, D. Alexandraki, G. Antoine, R. Anwar J.P.G. Ballesta, P. Benit, et al. 1992. The complete DNA sequence of yeast chromosome III. Nature 357: 38-46.

Pimpinelli, S., M. Berloco, L. Fanti, P. Dimitri, S. Bonaccorsi, E. Marchetti, R. Caizzi, C. Caggese, and M. Gatti. 1995. Transposable elements are stable structural components of Drosophila melanogaster heterochromatin. Proc. Natl. Acad. Sci. 92: 3804-3808.

Rine, J. and I. Herskowitz. 1987. Four genes responsible for a position effect on expression from $H M L$ and $H M R$ in Saccharomyces cerevisiae. Genetics 116: 9-22.

Roth, S. 1995. Chromatin-mediated transcriptional repression in yeast. Curr. Opin. Genet. Dev. 5: 168-173.

Rymond, B.C. and M. Rosbash. 1992. Yeast pre-mRNA splicing. In The molecular and cellular biology of the yeast Saccharomyces (ed. J.R. Broach, J.R. Pringle, and E.W. Jones), pp. 143-192. Cold Spring Harbor Laboratory Press, Cold Spring Harbor, NY.

Sandmeyer, S.B., L.J. Hansen, and D.L. Chalker. 1990. Integration specificity of retrotransposons and retroviruses. Annu. Rev. Genet. 24: 491-518.

Sharon, G., T.J. Burkett, and D.J. Garfinkel. 1994. Efficient homologous recombination of Tyl element cDNA when integration is blocked. Mol. Cell. Biol. 14: 6540-6551.

Thompson, J.S., X. Ling, and M. Grunstein. 1994. Histone H3 amino terminus is required for telomeric and silent mating locus repression in yeast. Nature 369: 245-247.

Voytas, D.F. and F.M. Ausubel. 1988. A copia-like transposable element family in Arabidopsis thaliana. Nature 336: 242244.

Voytas, D.F. and J.D. Boeke. 1992. Yeast retrotransposon revealed. Nature 358: 717 .

. 1993. Yeast retrotransposons and tRNAs. Trends Genet. 9: $421-427$.

Weinstock, K.G., M.F. Mastrangelo, T.J. Burkett, D.J. Garfinkel, and J.N. Strathern. 1990. Multimeric arrays of the yeast retrotransposon Ty1. Mol. Cell. Biol. 10: 2882-2892.

Xiong, Y. and T.H. Eickbush. 1990. Origin and evolution of retroelements based upon their reverse transcriptase sequences. $E M B O J$. 9: 3353-3362.

Yoshioka, K., H. Honma, M. Zushi, S. Kondo, S. Togashi, T. Miyake, and T. Shiba. 1990. Virus-like particle formation of Drosophila copia through autocatalytic processing. EMBO $/$. 9: 535-541.

Zou, S., D.A. Wright, and D.F. Voytas. 1995. The Saccharomyces Ty 5 retrotransposon family is associated with origins of DNA replication at the telomeres and the silent mating locus HMR. Proc. Natl. Acad. Sci. 92: 920-924. 


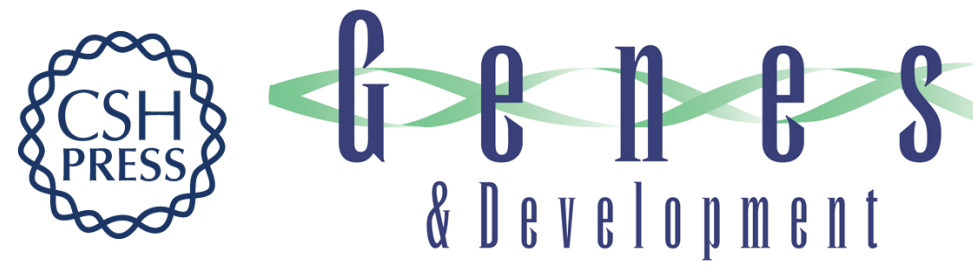

\section{The Saccharomyces retrotransposon Ty5 integrates preferentially into regions of silent chromatin at the telomeres and mating loci.}

S Zou, N Ke, J M Kim, et al.

Genes Dev. 1996, 10:

Access the most recent version at doi:10.1101/gad.10.5.634

References This article cites 52 articles, 26 of which can be accessed free at: http://genesdev.cshlp.org/content/10/5/634.full.html\#ref-list-1

License

Email Alerting

Service

Receive free email alerts when new articles cite this article - sign up in the box at the top right corner of the article or click here.

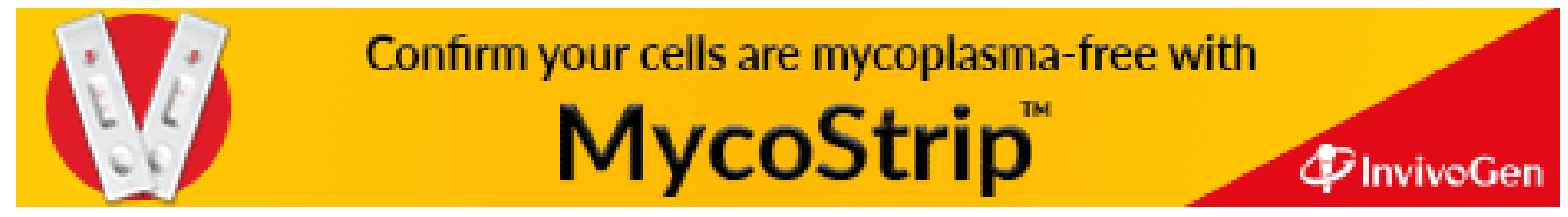

